\title{
Two draft genome sequences of Pseudomonas jessenii strains isolated from a copper contaminated site in Denmark
}

\author{
Yanan Qin', Dan Wang ${ }^{1,2}$, Kristian K. Brandt ${ }^{1}$ and Christopher Rensing ${ }^{3,4^{*}}$ (D)
}

\begin{abstract}
Pseudomonas jessenii C2 and Pseudomonas jessenii H16 were isolated from low-Cu and high-Cu industrially contaminated soil, respectively. P. jessenii H16 displayed significant resistance to copper when compared to P. jessenii C2. Here we describe genome sequences and interesting features of these two strains. The genome of $P$. jessenii $C 2$ comprised 6,420,113 bp, with 5814 protein-coding genes and 67 RNA genes. P. jessenii H16 comprised 6,807,788 bp, with 5995 protein-coding genes and 70 RNA genes. Of special interest was a specific adaptation to this harsh copper-contaminated environment as $P$. jessenii H16 contained a novel putative copper resistance genomic island (Gl) of around 50,000 bp.
\end{abstract}

Keywords: Pseudomonas jessenii, Comparative genomics, Copper resistance

\section{Introduction}

Copper is an essential micronutrient in most organisms and required as a co-factor in biological processes such as redox reactions (electron transport, oxidative respiration, denitrification) [1, 2]. However, at higher concentrations copper will become toxic and inhibit or kill cells. Therefore, microorganisms have developed sophisticated copper homeostasis and resistance mechanisms in order to maintain the normal cellular copper supply to essential cuproenzymes while avoiding copper poisoning $[3,4]$. Some highly copper resistant microorganisms have attracted great interests due to potential biotechnological applications in bio-mining and bioremediation of environments contaminated with copper [5].

Pseudomonas spp. are ubiquitous inhabitants of soil, water and plant surfaces belonging to the Gammaproteobacteria. Pseudomonas spp. has an exceptional capacity to produce a wide variety of secondary metabolites, including antibiotics that are toxic to plant pathogens [6, 7]. Pseudomonas jessenii was also found to be an important rhizobacterium conferring protection against a number of soilborne plant pathogens [8]. P. jessenii $\mathrm{C} 2$ and $P$. jessenii $\mathrm{H} 16$ were

\footnotetext{
* Correspondence: rensing@iue.ac.cn

${ }^{3}$ College of Resources and the Environment, Fujian Agriculture and Forestry University, Fuzhou, China

${ }^{4}$ J. Craig Venter Institute, La Jolla, CA, USA

Full list of author information is available at the end of the article
}

isolated from low- $\mathrm{Cu}$ soil and high-Cu soil from an abandoned wood impregnation site in Hygum, Denmark, respectively [9]. The Hygum site was contaminated with copper sulfate from 1911 to 1924, then the area was cultivated until 1993 and has been a fallow field since then $[9,10]$. P. jessenii H16 was able to grow in medium containing high concentrations of copper, whereas $P$. jessenii C2 was sensitive to high copper concentrations. Here, we present the genome sequences, a brief characterization and annotation of $P$. jessenii $\mathrm{C} 2$ and $P$. jessenii $\mathrm{H} 16$.

\section{Organism information Classification and features}

A highly copper contaminated high-Cu soil and a corresponding low-Cu soil were collected $(0-20 \mathrm{~cm}$ depth) from a well-described $\mathrm{Cu}$ gradient field site in Hygum, Denmark. The high-Cu site was contaminated almost exclusively with $\mathrm{CuSO}_{4}$ more than 90 years ago [9]. The adjacent low-Cu control site was located just outside the contaminated area and had been subjected to the same land use for more than 80 years. The low- $\mathrm{Cu}$ and high- $\mathrm{Cu}$ soil had similar physicochemical characteristics except for their total $\mathrm{Cu}$ contents of 21 and $3172 \mathrm{mg} \mathrm{kg}^{-1}$, respectively $[9,11]$. Bacteria were isolated from replicated soil subsamples $(n=3)$ and diluted, spread-plated on Pseudomonas-selective Gould's S1 agar [11]. For each dilution series, 30 colonies emerging after two days at $25{ }^{\circ} \mathrm{C}$ were selected and isolated in pure 
culture by repeated plating [11]. Two of the resulting isolates were selected for further study. P. jessenii H16 was able to grow at high concentration of $\mathrm{Cu}(2 \mathrm{mM})$ on onetenth strength LB agar, whereas $P$. jessenii $\mathrm{C} 2$ only grew with up to $0.125 \mathrm{mM} \mathrm{Cu}$.

Strain C2 and H16 were both Gram-reaction negative. Cells of strain $\mathrm{C} 2$ and $\mathrm{H} 16$ were rod shaped with rounded ends and motile. The cells of $\mathrm{C} 2$ were $2.12-2.45 \mu \mathrm{m}$ (mean, $2.28 \mu \mathrm{m}$ ) in length compared to $0.49-0.62 \mu \mathrm{m}$ (mean, $0.55 \mu \mathrm{m}$ ) in width (Fig. 1a). The cells of H16 were $1.95-2.38 \mu \mathrm{m} \times 0.42-0.57 \mu \mathrm{m}$ in size (Fig. 1b). No Sporulation was observed for both strains. The colonies were white and translucent on Gould's S1 agar medium. Growth occurred aerobically at $4-37{ }^{\circ} \mathrm{C}$, and optimal growth was observed at $30^{\circ} \mathrm{C}, \mathrm{pH} 7.0$ for strain $\mathrm{C} 2$. Strain $\mathrm{H} 16$ preferred $\mathrm{pH} 6.7$, at $30{ }^{\circ} \mathrm{C}$ for optimal growth. Both strains grew in $0-4 \%(\mathrm{w} / \mathrm{v}) \mathrm{NaCl}$ (Tables 1 and 2).

\section{Chemotaxonomy}

Fatty acid analyses were performed by the Identification Service of the DSMZ, Braunschweig, Germany [12]. The fatty acid profiles were similar when comparing strains $\mathrm{C} 2$ and H16. The major fatty acids of the two strains showed as follows: $\mathrm{C}_{16:} \omega 7 \mathrm{c}$ and/or iso- $\mathrm{C}_{15 \text { : } 0} 2-\mathrm{OH}(36.4 \%$ in $P$. jessenii $\mathrm{C} 2$ and $40.1 \%$ in P. jessenii $\mathrm{H} 16) ; \mathrm{C}_{18}: 1 \omega 7 c$ (15.3\% in P. jessenii $\mathrm{C} 2$ and $10.8 \%$ in P. jessenii $\mathrm{H} 16)$ and $\mathrm{C}_{16: 0}$ (28.8 \% in P. jessenii $\mathrm{C} 2$ and $34.6 \%$ P. jessenii $\left.\mathrm{H} 16\right)$.

Biochemical properties were tested using API 20NE (BioMérieux) for Strains C2 and H16. In the API 20NE system, positive reactions for both strains were observed for nitrate reduction and production of arginine dihydrolase; negative reactions were observed for indole production, urease activity, Lysine and ornithine decarboxylase and gelatin hydrolysis (Additional file 1: Table S1). Strain C2 assimilated d-glucose, d-melibiose, d-sucrose, dmannitol, l-rhamnose, inositol, trehalose, d-lyxose and larabinose, but not sorbitol. Strain H16 could utilize dglucose, d-melibiose, d-sucrose, d-mannitol, trehalose, d- lyxose, l-arabinose and inostitol as carbon sources, but not, 1-rhamnose and sorbitol (Additional file 1: Table S1).

\section{S rRNA gene analysis}

Comparative $16 \mathrm{~S}$ rRNA gene sequence analysis using the EzTaxon database [13] indicated that strain $\mathrm{C} 2$ and $\mathrm{H} 16$ were both most closely related to $P$. jessenii CIP $105275^{\mathrm{T}}$ (GenBank accession no. AF068259) with sequence similarities of 99.87 and $99.14 \%$, respectively. Phylogenetic analysis was performed using the $16 \mathrm{~S}$ rRNA gene sequences of strains C2, H16 and related species. Sequences were aligned and phylogenic trees were constructed using Maximum Likelihood method implemented in MEGA version 6 [14]. The resultant tree topologies were evaluated by bootstrap analyses with 1000 random samplings (Fig. 2).

\section{Genome sequencing information} Genome project history

Next-generation shotgun-sequencing was performed at the Beijing Genomics Institute (BGI, Shenzhen). The whole genome shotgun project of $P$. jessenii $\mathrm{C} 2$ and $P$. jessenii $\mathrm{H} 16$ has been deposited at DDBJ/EMBL/GenBank under the accession numbers JSAK00000000 and JSAL00000000. The version described in this paper is the first version. A summary of the project and the Minimum Information about a Genome Sequence [15] are shown in Table 3.

\section{Growth conditions and genomic DNA preparation}

$P$. jessenii $\mathrm{C} 2$ and $P$. jessenii $\mathrm{H} 16$ were aerobically cultivated on Pseudomonas-selective Gould's S1 agar at $28{ }^{\circ} \mathrm{C}$ [16]. Total genomic DNA was extracted using Puregene Yeast/Bact Kit according to the manufacturer's instructions (QIAGEN). The quantity of the genomic DNA was determined by Qubit $^{\circ}$ fluorometer (Invitrogen, CA, USA) with Qubit dsDNA BR Assay kit (Invitrogen, CA, USA) and amounted to $55 \mathrm{ng} / \mu \mathrm{L}$ of DNA for $P$. jessenii $\mathrm{C} 2$ and $48.2 \mathrm{ng} / \mu \mathrm{L}$ of DNA for $P$. jessenii H16.

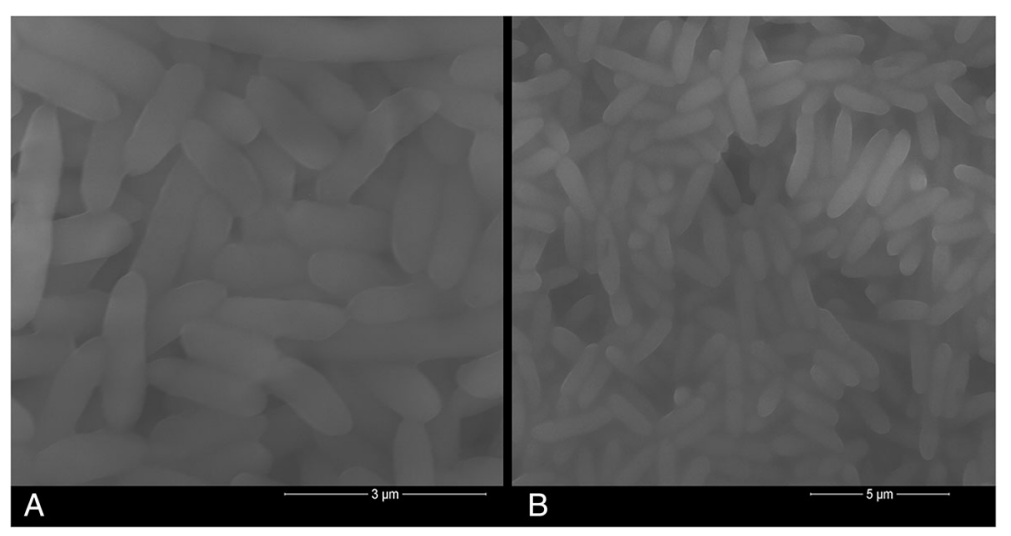

Fig. 1 Micrograph of Pseudomonas jessenii C2 and H16 obtained by scanning electron microscopy. a Pseudomonas jessenii C2. b Pseudomonas jessenii H16 
Table 1 Classification and general features of P.jessenii C2 according to the MIGS recommendations [15]

\begin{tabular}{|c|c|c|c|}
\hline MIGS ID & Property & Term & $\begin{array}{l}\text { Evidence } \\
\text { code }^{a}\end{array}$ \\
\hline & Classification & Domain Bacteria & TAS [40] \\
\hline & & Phylum Proteobacteria & TAS [41] \\
\hline & & Class Gammaproteobacteria & $\begin{array}{l}\text { TAS [42, } \\
43]\end{array}$ \\
\hline & & Order Pseudomonadales & TAS [44] \\
\hline & & Family Pseudomonadaceae & TAS [45] \\
\hline & & Genus Pseudomonas & $\begin{array}{l}\text { TAS [46, } \\
47]\end{array}$ \\
\hline & & Species $P$. jessenii & TAS [48] \\
\hline & & strain: C2 & IDA \\
\hline & Gram stain & Negative & IDA \\
\hline & Cell shape & Rod-shaped & IDA \\
\hline & Motility & Motile & IDA \\
\hline & Sporulation & Non-sporulating & IDA \\
\hline & $\begin{array}{l}\text { Temperature } \\
\text { range }\end{array}$ & $4-37^{\circ} \mathrm{C}$ & IDA \\
\hline & $\begin{array}{l}\text { Optimum } \\
\text { temperature }\end{array}$ & $30^{\circ} \mathrm{C}$ & IDA \\
\hline & Optimum pH & 7.0 & IDA \\
\hline & Carbon source & $\begin{array}{l}\text { d-glucose, d-melibiose, d-sucrose, } \\
\text { d-mannitol, L-rhamnose, inositol, } \\
\text { trehalose, d-lyxose,L-arabinose }\end{array}$ & IDA \\
\hline MIGS-6 & Habitat & soil & IDA \\
\hline MIGS-6.3 & Salinity & $0-4 \%$ & IDA \\
\hline MIGS-22 & $\begin{array}{l}\text { Oxygen } \\
\text { requirement }\end{array}$ & Aerobic & IDA \\
\hline MIGS-15 & $\begin{array}{l}\text { Biotic } \\
\text { relationship }\end{array}$ & Free-living & IDA \\
\hline MIGS-14 & Pathogenicity & Non-pathogen & NAS \\
\hline MIGS-4 & $\begin{array}{l}\text { Geographic } \\
\text { location }\end{array}$ & Hygum, Denmark & IDA \\
\hline MIGS-5 & $\begin{array}{l}\text { Sample } \\
\text { collection }\end{array}$ & May 2013 & IDA \\
\hline MIGS-4.1 & Latitude & $55^{\circ} 46^{\prime} 25^{\prime \prime} \mathrm{N}$ & IDA \\
\hline MIGS-4.2 & Longitude & $9^{\circ} 25^{\prime} 52^{\prime \prime} \mathrm{E}$ & IDA \\
\hline
\end{tabular}

${ }^{a}$ Evidence codes - IDA inferred from direct assay, TAS traceable author statement (i.e., a direct report exists in the literature), NAS non-traceable author statement (i.e., not directly observed for the living, isolated sample, but based on a generally accepted property for the species, or anecdotal evidence). These evidence codes are from the Gene Ontology project [49]. If the evidence is IDA, the property was directly observed by the authors

\section{Genome sequencing and assembly}

The genome sequence of $P$. jessenii $\mathrm{H} 16$ and $P$. jessenii C2 was determined by BGI using the Illumina Hiseq2000 with a 500 bp library constructed [17], generating 1.09 gigabytes of DNA sequence with an average coverage of $\sim 160$ fold and $\sim 170$ fold; yielding 1,205,9244 and $1,203,8756$ paired-end reads with a 90-bp read
Table 2 Classification and general features of P.jessenii H16 according to the MIGS recommendations [15]

\begin{tabular}{|c|c|c|c|}
\hline MIGS ID & Property & Term & $\begin{array}{l}\text { Evidence } \\
\text { code }^{\mathrm{a}}\end{array}$ \\
\hline & Classification & Domain Bacteria & TAS [40] \\
\hline & & Phylum Proteobacteria & TAS [41] \\
\hline & & Class Gammaproteobacteria & $\begin{array}{l}\text { TAS }[42, \\
43]\end{array}$ \\
\hline & & Order Pseudomonadales & TAS [44] \\
\hline & & Family Pseudomonadaceae & TAS [45] \\
\hline & & Genus Pseudomonas & $\begin{array}{l}\text { TAS [46, } \\
47]\end{array}$ \\
\hline & & Species $P$. jessenii & TAS [48] \\
\hline & & strain: H16 & IDA \\
\hline & Gram stain & Negative & IDA \\
\hline & Cell shape & Rod-shaped & IDA \\
\hline & Motility & Motile & IDA \\
\hline & Sporulation & Non-sporulating & IDA \\
\hline & $\begin{array}{l}\text { Temperature } \\
\text { range }\end{array}$ & $4-37^{\circ} \mathrm{C}$ & IDA \\
\hline & $\begin{array}{l}\text { Optimum } \\
\text { temperature }\end{array}$ & $30^{\circ} \mathrm{C}$ & IDA \\
\hline & Optimum pH & 6.7 & IDA \\
\hline & Carbon source & $\begin{array}{l}\text { d-glucose, d-melibiose, d-sucrose, } \\
\text { d-mannitol, trehalose, d-lyxose, } \\
\text { L-arabinose,inostitol }\end{array}$ & IDA \\
\hline MIGS-6 & Habitat & Copper contaminated soil & IDA \\
\hline MIGS-6.3 & Salinity & $0-4 \%$ & IDA \\
\hline MIGS-22 & $\begin{array}{l}\text { Oxygen } \\
\text { requirement }\end{array}$ & Aerobic & IDA \\
\hline MIGS-15 & $\begin{array}{l}\text { Biotic } \\
\text { relationship }\end{array}$ & Free-living & IDA \\
\hline MIGS-14 & Pathogenicity & Non-pathogen & NAS \\
\hline MIGS-4 & $\begin{array}{l}\text { Geographic } \\
\text { location }\end{array}$ & Hygum, Denmark & IDA \\
\hline MIGS-5 & $\begin{array}{l}\text { Sample } \\
\text { collection }\end{array}$ & May 2013 & IDA \\
\hline MIGS-4.1 & Latitude & $55^{\circ} 46^{\prime} 25^{\prime \prime} \mathrm{N}$ & IDA \\
\hline MIGS-4.2 & Longitude & $9^{\circ} 25^{\prime} 52^{\prime \prime} \mathrm{E}$ & IDA \\
\hline
\end{tabular}

aEvidence codes - IDA inferred from direct assay, TAS traceable author statement (i.e., a direct report exists in the literature), NAS non-traceable author statement (i.e., not directly observed for the living, isolated sample, but based on a generally accepted property for the species, or anecdotal evidence). These evidence codes are from the Gene Ontology project [49]. If the evidence is IDA, the property was directly observed by the authors

length, respectively. The resulting sequence data was quality assessed, trimmed, and assembled de novo as described previously [18] using CLCBio Genomic Workbench 7.0 (CLCBio, Denmark). P. jessenii H16 generated 78 contigs with an n50 value of 279,014 bp. P. jessenii $\mathrm{C} 2$ generated 64 contigs with an $\mathrm{n} 50$ value of $224,893 \mathrm{bp}$. 


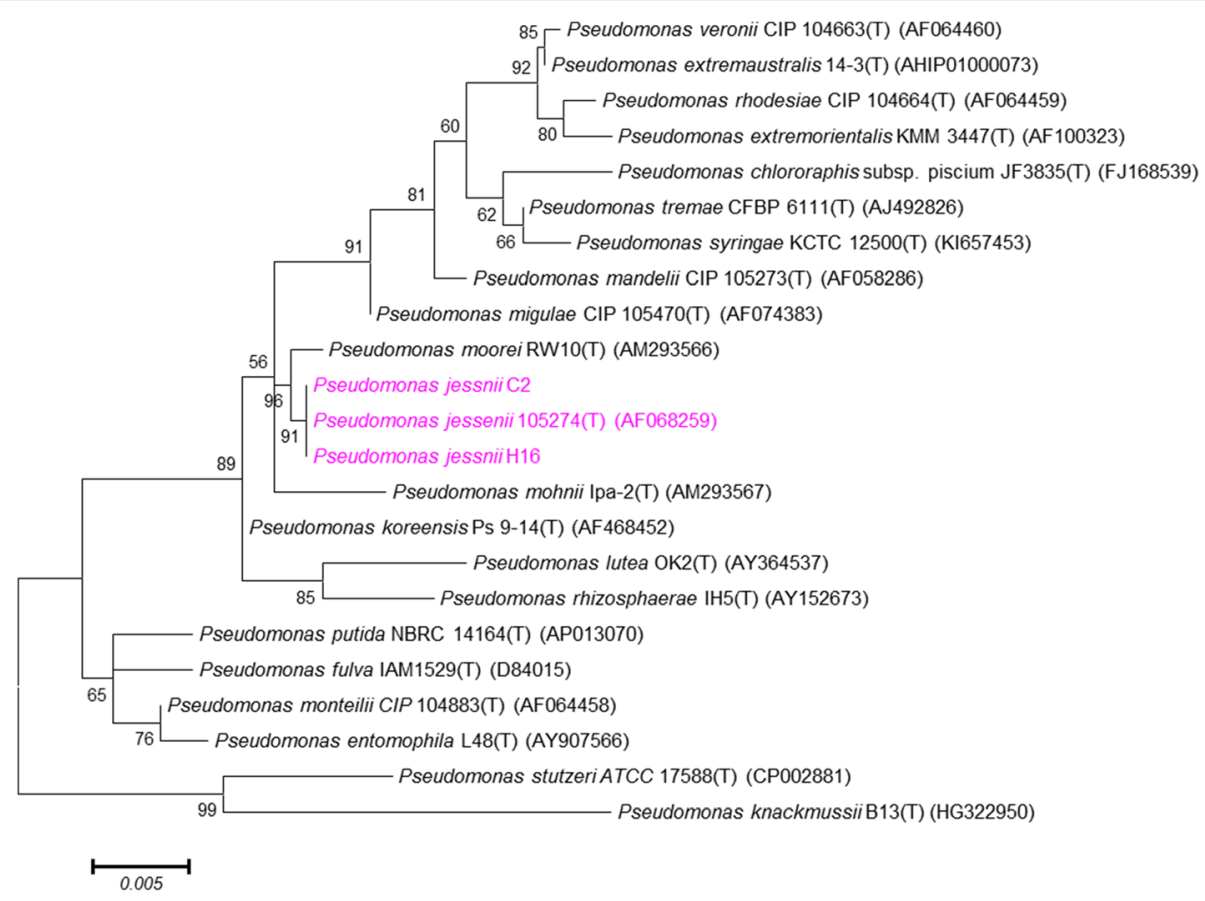

Fig. 2 Phylogenetic tree of $P$. jessenii $C 2$ and $P$. jessenii $\mathrm{H} 16$ relative to type strains within the genus Pseudomonas. The strains and their corresponding GenBank accession numbers of $16 \mathrm{~S}$ rRNA genes are displayed in parentheses. The sequences were aligned using Clustal W, and the maximum likelihood tree was constructed based on Jukes-Cantor model by using MEGA6 [14]. Bootstrap values above $50 \%$ are shown obtained from 1000 bootstrap replications. Bar 0.005 substitutions per nucleotide position

\section{Genome annotation}

The genes in the assembled genome were predicted based on the RAST database [19]. The predicted ORFs were annotated by searching clusters of orthologous groups [20] using the SEED database [21]. RNAmmer 1.2 [22] and tRNAscanSE 1.23 [23] were used to identify rRNA and tRNA genes, respectively.

\section{Genome properties}

$P$. jessenii $\mathrm{C} 2$ contained 6,420,113 bp with a $\mathrm{G}+\mathrm{C}$ content of $59.83 \%, 5881$ predicted genes, 5814 were proteincoding genes, 63 tRNA genes and 4 rRNA genes. In total, 5179 genes were assigned to biological functions and 635 were annotated as hypothetical proteins. P. jessenii $\mathrm{H} 16$ contained $6,807,788 \mathrm{bp}$, with a GC content of $59.02 \%$,

Table 3 Project information

\begin{tabular}{|c|c|c|c|}
\hline MIGS ID & Property & Term & \\
\hline MIGS 31 & Finishing quality & High-quality draft & High-quality draft \\
\hline MIGS-28 & Libraries used & One paired-end Illumina library & One paired-end Illumina library \\
\hline MIGS 29 & Sequencing platforms & Illumina HiSeq 2000 & Illumina HiSeq 2000 \\
\hline MIGS 31.2 & Fold coverage & $170 \times$ & $160 \times$ \\
\hline MIGS 30 & Assemblers & $\begin{array}{l}\text { CLC Genomics } \\
\text { Workbench, version7.0.4 }\end{array}$ & $\begin{array}{l}\text { CLC Genomics } \\
\text { Workbench, version7.0.4 }\end{array}$ \\
\hline \multirow[t]{6}{*}{ MIGS 32} & Gene calling method & Glimmer 3.0 & Glimmer 3.0 \\
\hline & Locus Tag & NL64 & RY26 \\
\hline & Genbank ID & JSAK00000000.1 & JSAL00000000.1 \\
\hline & GenBank Date of Release & $2014 / 12 / 17$ & 2014/12/17 \\
\hline & GOLD ID & Gp0157184 & Gp0157185 \\
\hline & BIOPROJECT & PRJNA264019 & PRJNA264019 \\
\hline \multirow[t]{2}{*}{ MIGS 13} & Source Material Identifier & $\mathrm{HC}-\mathrm{Cu} 02$ & HC_Cu16 \\
\hline & Project relevance & Low-Cu soil & Copper contaminated soil \\
\hline
\end{tabular}


Table 4 Genome statistics

\begin{tabular}{|c|c|c|c|c|}
\hline \multirow[t]{2}{*}{ Attribute } & \multicolumn{2}{|c|}{ P. jessenii C2 } & \multicolumn{2}{|c|}{ P. jessenii $\mathrm{H} 16$} \\
\hline & Value & $\%$ of total & Value & $\%$ of total \\
\hline Genome size (bp) & $6,420,113$ & 100.00 & $6,807,788$ & 100.00 \\
\hline DNA coding (bp) & $5,484,120$ & 85.42 & $5,835,906$ & 85.72 \\
\hline DNA G+C (bp) & $3,851,154$ & 59.83 & $4,017,956$ & 59.02 \\
\hline DNA scaffolds & 64 & - & 78 & - \\
\hline Total genes & 5881 & 100.00 & 6065 & 100.00 \\
\hline Protein coding genes & 5814 & 98.86 & 5995 & 98.85 \\
\hline RNA genes & 67 & 1.14 & 70 & 1.15 \\
\hline \multicolumn{5}{|l|}{ Pseudo genes } \\
\hline Genes with function prediction & 5179 & 88.06 & 5344 & 88.11 \\
\hline Genes assigned to COGs & 4314 & 73.75 & 4354 & 71.79 \\
\hline Genes with Pfam domains & 3595 & 61.13 & 3587 & 59.14 \\
\hline Genes with signal peptides & 510 & 8.67 & 537 & 8.85 \\
\hline Genes with transmembrane helices & 1260 & 21.42 & 1343 & 22.14 \\
\hline CRISPR repeats & 38 & - & 11 & - \\
\hline
\end{tabular}

Table 5 Number of genes associated with general COG functional categories

\begin{tabular}{|c|c|c|c|c|c|}
\hline \multirow[b]{2}{*}{ Code } & \multicolumn{2}{|c|}{ P. jessenii C2 } & \multicolumn{2}{|c|}{ P. jessenii H16 } & \multirow[b]{2}{*}{ Description } \\
\hline & Value & $\%^{\mathrm{a}}$ & Value & $\%^{\mathrm{a}}$ & \\
\hline J & 183 & 3.14 & 186 & 3.10 & Translation, ribosomal structure and biogenesis \\
\hline A & 1 & 0.02 & 2 & 0.03 & RNA processing and modification \\
\hline K & 425 & 7.31 & 425 & 7.09 & Transcription \\
\hline$L$ & 147 & 2.53 & 135 & 2.25 & Replication, recombination and repair \\
\hline B & 2 & 0.34 & 3 & 0.05 & Chromatin structure and dynamics \\
\hline $\mathrm{D}$ & 35 & 0.60 & 35 & 0.58 & Cell cycle control, Cell division, chromosome partitioning \\
\hline V & 59 & 1.01 & 57 & 0.95 & Defense mechanisms \\
\hline $\mathrm{T}$ & 368 & 6.33 & 389 & 6.49 & Signal transduction mechanisms \\
\hline M & 239 & 4.11 & 282 & 4.70 & Cell wall/membrane biogenesis \\
\hline $\mathrm{N}$ & 128 & 2.20 & 135 & 2.25 & Cell motility \\
\hline U & 119 & 2.05 & 128 & 2.14 & Intracellular trafficking and secretion \\
\hline $\mathrm{O}$ & 175 & 3.01 & 168 & 2.80 & Posttranslational modification, protein turnover, chaperones \\
\hline $\mathrm{C}$ & 312 & 5.37 & 278 & 4.64 & Energy production and conversion \\
\hline G & 219 & 3.77 & 247 & 4.12 & Carbohydrate transport and metabolism \\
\hline $\mathrm{E}$ & 515 & 8.86 & 497 & 8.29 & Amino acid transport and metabolism \\
\hline $\mathrm{F}$ & 85 & 1.46 & 99 & 1.65 & Nucleotide transport and metabolism \\
\hline $\mathrm{H}$ & 177 & 3.04 & 193 & 3.22 & Coenzyme transport and metabolism \\
\hline I & 237 & 4.08 & 194 & 3.24 & Lipid transport and metabolism \\
\hline$P$ & 300 & 5.16 & 286 & 4.77 & Inorganic ion transport and metabolism \\
\hline Q & 142 & 2.44 & 129 & 2.15 & Secondary metabolites biosynthesis, transport and catabolism \\
\hline $\mathrm{R}$ & 532 & 9.15 & 572 & 9.54 & General function prediction only \\
\hline S & 444 & 7.64 & 451 & 7.52 & Function unknown \\
\hline- & 970 & 16.68 & 1104 & 18.42 & Not in COGs \\
\hline
\end{tabular}


6065 predicted genes, and 5995 were protein-coding genes, 65 tRNA and 5 rRNA genes. Among the protein coding genes 5344 were assigned to biological functions, while 651 were annotated as hypothetical proteins. The properties and statistics of those two genomes are summarized in Table 4. The distribution of genes into COG functional categories is presented in Table 5 and Fig. 3.

\section{Insights into the genome}

Genes conferring resistances to heavy metals in the two studied strains are listed in Table 6. Copper efflux from the cytosol is mediated by the $\mathrm{P}_{1 \mathrm{~B}}$-type ATPase family, which is highly conserved from bacteria to humans [24]. Both $P$. jessenii $\mathrm{C} 2$ and $P$. jessenii $\mathrm{H} 16$ contained genes encoding a copper-transporting $\mathrm{P}_{1 \mathrm{~B}}$-type ATPase (CopA) with conserved CPCALG motif [25], a copper-responsive metalloregulatory protein $\mathrm{CueR}$, and the multicopper oxidase CueO. In addition, one additional gene encoding a $\mathrm{Cu}$ ${ }^{+}$-ATPase is present on the genome of $P$. jessenii $\mathrm{H} 16$ as part of the GI discussed later. P. jessenii H16 also contained $\mathrm{ccoI}$ encoding a $\mathrm{Cu}^{+}$-ATPase catalyzing a slower rate of efflux for copper insertion into cytochrome c oxidase [26]. The presence of a cop operon, comprising copABCDRS had been reported in related P.fluorescens SBW25 and P.putida KT2440 [27, 28]. Both P. jessenii strains contained $\operatorname{cop} C D R S$ probably encoding proteins

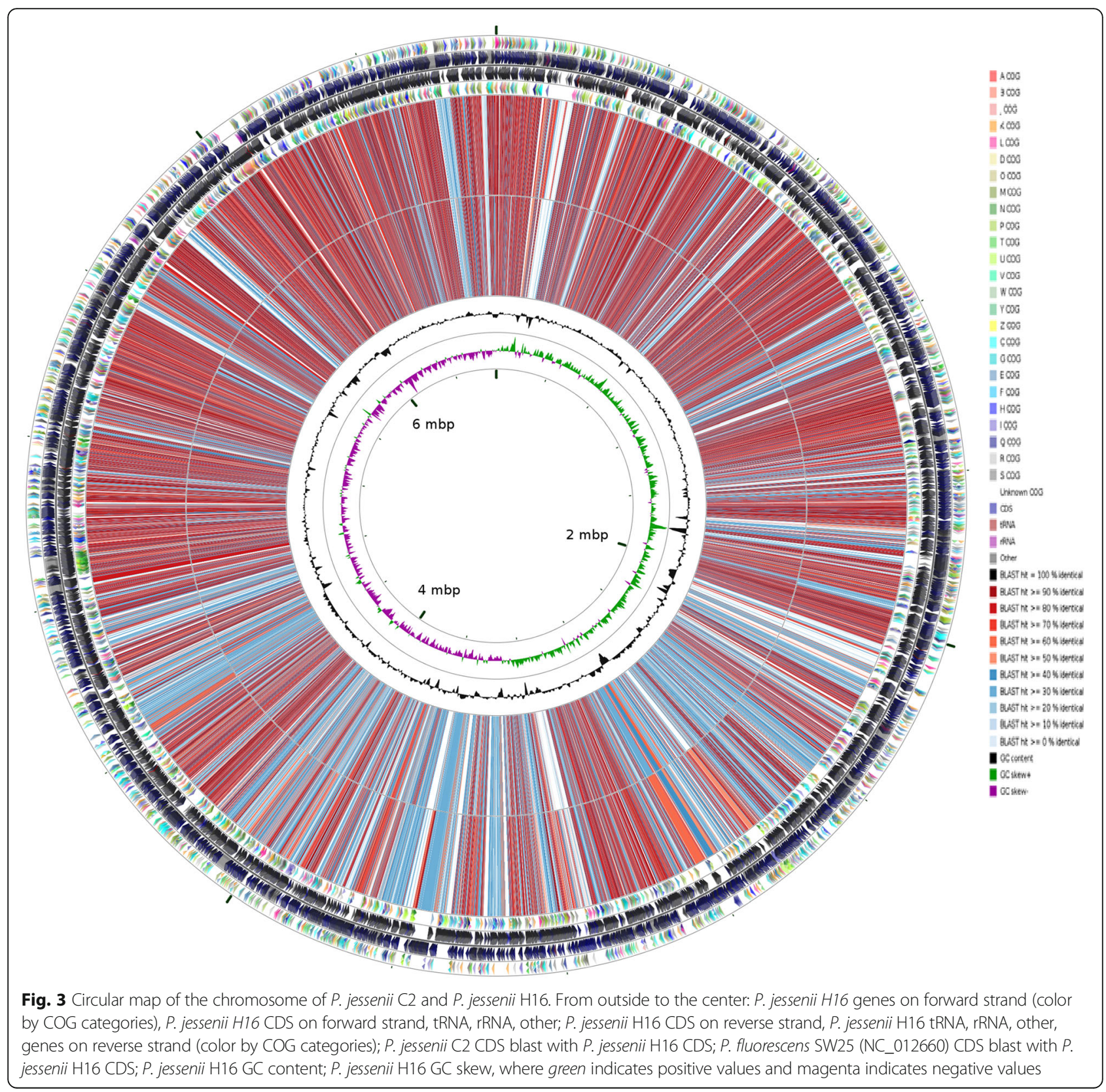


Table 6 P.jessenii C2 and P.jessenii H16 genes related to heavy metal resistance

\begin{tabular}{|c|c|c|c|c|}
\hline \multicolumn{2}{|c|}{ P.jessenii C2 } & \multicolumn{2}{|c|}{ P.jessenii $\mathrm{H} 16$} & \multirow[b]{2}{*}{ Predicted function } \\
\hline $\begin{array}{l}\text { Protein } \\
\text { id }\end{array}$ & Size/aa & $\begin{array}{l}\text { Protein } \\
\text { id }\end{array}$ & Size/aa & \\
\hline KII28258 & 513 & KII28679 & 459 & Multicopper oxidase CueO-1 \\
\hline KII31612 & 122 & KII28987 & 121 & Copper resistance protein CopC \\
\hline KII31613 & 282 & KII28988 & 286 & Copper resistance protein CopD-1 \\
\hline KII30013 & 133 & KII32596 & 138 & $\begin{array}{l}\mathrm{Cu}(\mathrm{l}) \text {-responsive transcriptional } \\
\text { regulator CopR }\end{array}$ \\
\hline KII30014 & 798 & KIII32595 & 798 & $\begin{array}{l}\text { Copper-translocating P-type } \\
\text { ATPase CopA-1 }\end{array}$ \\
\hline KII30016 & 66 & KII32593 & 66 & Copper resistance protein CopZ \\
\hline KII37329 & 149 & KII29565 & 149 & Metal-binding protein CopG-1 \\
\hline KII33434 & 179 & KII28041 & 179 & Copper tolerance protein \\
\hline KII33435 & 227 & KII28042 & 227 & Copper response regulator CusR-1 \\
\hline KII33436 & 450 & KII28043 & 450 & $\begin{array}{l}\text { Copper sensor histidine kinase } \\
\text { CusS-1 }\end{array}$ \\
\hline KII34384 & 759 & KIII5062 & 770 & $\begin{array}{l}\text { Lead, cadmium, zinc and mercury } \\
\text { transporting ATPase }\end{array}$ \\
\hline KII29503 & 231 & KII36596 & 231 & Arsenic resistance protein $\mathrm{ArsH}$ \\
\hline KII29504 & 157 & KII36597 & 157 & Arsenate reductase ArsC \\
\hline KII29505 & 428 & KII36598 & 116 & $\begin{array}{l}\text { Arsenical resistance operon } \\
\text { repressor ArsR }\end{array}$ \\
\hline KII29506 & 116 & KIII6460 & 428 & $\begin{array}{l}\text { Arsenical pump membrane } \\
\text { protein ArsB }\end{array}$ \\
\hline \multirow[t]{21}{*}{ KIII31669 } & 453 & KII30277 & 447 & Chromate transport protein ChrA \\
\hline & & KIII7024 & 798 & Cytochrome c oxidases \\
\hline & & KII37706 & 1047 & Cation transporter CusA \\
\hline & & KII37707 & 494 & RND transporter CusB \\
\hline & & KII37708 & 418 & $\begin{array}{l}\text { RND efflux outer membrane } \\
\text { protein CusC }\end{array}$ \\
\hline & & KII37709 & 312 & Copper resistance protein CopD-2 \\
\hline & & KII37710 & 462 & Copper sensor histidine kinase CusS-2 \\
\hline & & KII37711 & 231 & Copper response regulator CusR-2 \\
\hline & & KIII77713 & 178 & $\begin{array}{l}\text { Blue (type1) copper domain- } \\
\text { containing protein }\end{array}$ \\
\hline & & KII37893 & 676 & $\begin{array}{l}\text { Copper-translocating P-type } \\
\text { ATPase CopA-2 }\end{array}$ \\
\hline & & KII37715 & 642 & Multicopper oxidase CueO-2 \\
\hline & & KII37716 & 333 & Copper resistance protein CopB \\
\hline & & KII37717 & 155 & Metal-binding protein CopG-2 \\
\hline & & KII37719 & 321 & Cation transporter $\mathrm{CzCD}$ \\
\hline & & KII37721 & 436 & Nickel efflux system NrcA \\
\hline & & KIII77723 & 99 & Nickel resistance protein NcrB \\
\hline & & KII37733 & 116 & Mercuric transport protein MerT \\
\hline & & KIII77734 & 91 & Mercury transporter MerR \\
\hline & & KII37735 & 144 & Mercury transport protein MerC \\
\hline & & KII37736 & 560 & Mercuric reductase MerA \\
\hline & & KII37737 & 212 & Alkylmercury lyase MerB \\
\hline
\end{tabular}

responsible for copper uptake, however, only $P$. jessenii $\mathrm{H} 16$ also contained $\operatorname{cop} A B$ as part of the GI. Both $P$. jessenii $\mathrm{C} 2$ and $P$. jessenii $\mathrm{H} 16$ contain an arsenic resistance determinant $(\operatorname{ars} R B C H)$ [29] a gene involved in chromate resistance (chrA) [26] (Table 6). The two strains also contained genes encoding a multidrug efflux system MexEFOprN regulated by MexT and genes encoding DNA gyrase subunit $\mathrm{A}$ and $\mathrm{B}$, and topoisomerase subunit (IV) A and B $[30,31]$.

$P$. jessenii $\mathrm{H} 16$ contained an additional putative metal fitness/pathogenicity island when compared with $P$. jessenii C2. It encompasses about 50,000 bp beginning at a gene encoding a sulfur carrier protein (KII37703) and ending with genes encoding Tn7 transposition proteins (KII37740-KII37743). This potential pathogenicity/fitness island harbored several copper resistance determinants including the cus determinant encoding CusABCRS (KII37706-37708, KII37711-37712) involved in periplasmic copper detoxification [32, 33]. In addition, genes encoding the P-type ATPase CopA, the multicopper oxidase CueO and CopBDG (KII37893, KII37715, KII37716, KII37709, KII37717) could be identified (Fig. 4). We also predicted specific GI for both $P$. jessenii $\mathrm{H} 16$ and P. jessenii $\mathrm{C} 2$ using the IsfindViewer [34]. Based on the automatic prediction algorithm two putative regions (coordinates KII37706-KII37717, KII37721KII37737) were only identified in P. jessenii H16. Similar copper fitness islands could also be detected in P.extremaustralis 14-3b (AHIP00000000), isolated from a temporary pond in Antarctica; Pseudomonas sp.Ag1 (AKVH00000000) isolated from midguts of mosquitoes and P. fluorescens FH4 (AOHN00000000) [35-37]. This island also contained genes encoding the nickel efflux transporter NcrA (KII37721) and the transcriptional repressor NcrB (KII37723) [38]. Moreover, genes merTR$C A B$ (KII37733-37737) encoding a mercury-resistance determinant are present on this island [39]. Many of the various putative GI contain functions related to mobility such as integrases or mobile genetic elements (MGE) which includes transposons and IS elements. As shown in $P$. jessenii H16, these putative GI have conferred this strain with additional heavy metal resistance capability, which may be transferred to other bacteria via Tn7 transposons and are highly relevant for adaption to this specific copper contaminated niche.

\section{Conclusion}

The draft genome sequences of $P$. jessenii $\mathrm{C} 2$ isolated from low-Cu soil and $P$. jessenii $\mathrm{H} 16$ isolated from high$\mathrm{Cu}$ soil were determined and described here. H16 provided an insight into the genomic basis of the observed higher copper resistance when compared with $\mathrm{C} 2$. Based on analysis and characterization of the genome, $P$. jessenii H16 is predicted to be resistant to a number of heavy 

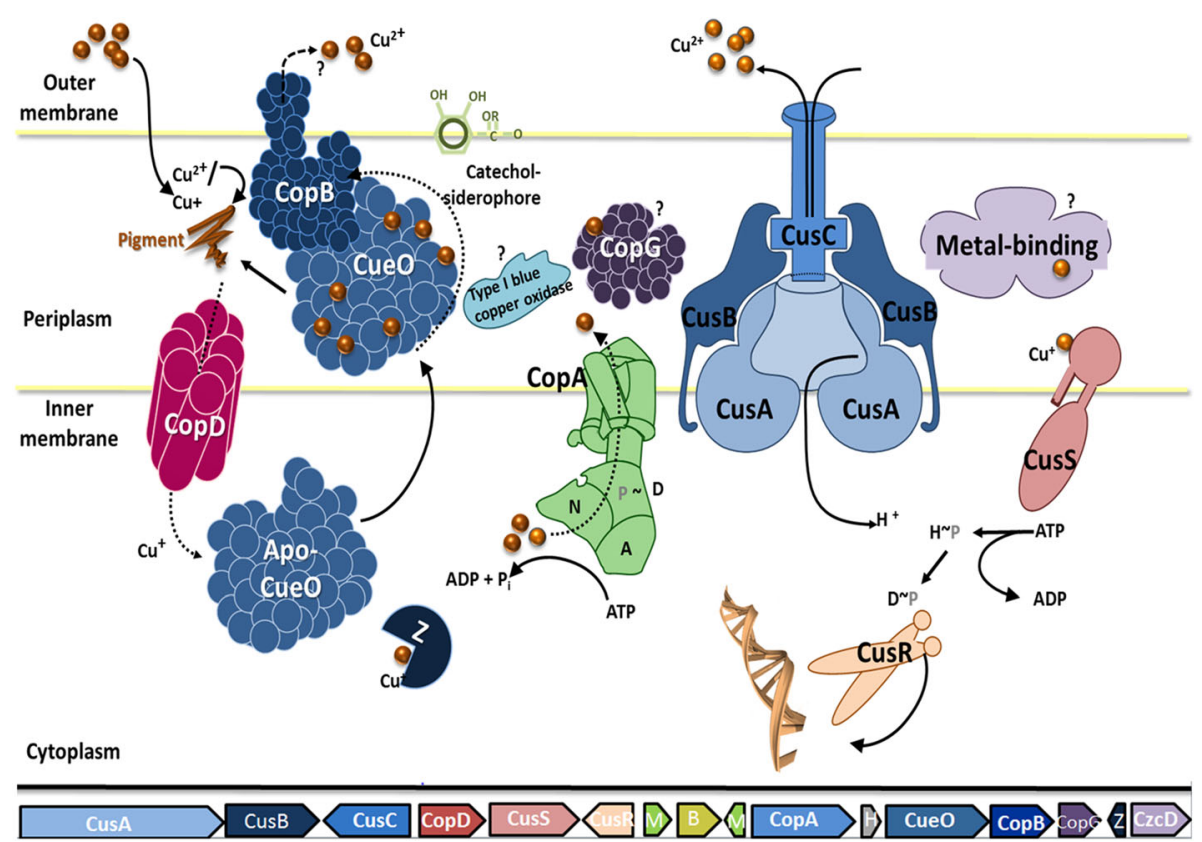

Fig. 4 Putative copper fitness/pathogenicity island in P.jessenii H16. Model of encoded proteins involved in copper resistance. CusA copper transporter, CusB RND transporter, CusC RND efflux outer membrane protein, CopD copper resistance protein, CusS-2 copper sensor histidine kinase, CusR-2 copper response regulator, CopA-2 copper-translocating P-type ATPase, CueO-2 multicopper oxidase, CopB copper resistance protei, CopG-2 metal-binding protein, $C z C D$ cation transporter, $B$ blue (type1) copper domain-containing protein CinA, $H$ hypothetical protein, $M$ putative metal-binding protein, $Z$ copper chaperone

metal(loid)s, such as $\mathrm{Hg}^{2+}, \mathrm{Ni}^{2+} \mathrm{Cr}^{2+}$ and $\mathrm{As}^{3+}$. Comparative genomic analysis of those two strains suggested acquisition of a fitness island encoding numerous genes involved in conferring resistance to $\mathrm{Cu}$ and other metals as an important adaptive mechanism enabling survival of $P$. jessenii $\mathrm{H} 16$ in its $\mathrm{Cu}$ contaminated habitat. Possibly, $P$. jessenii $\mathrm{H} 16$ may have potential for bioremediation of copper contamination environments.

\section{Additional file}

Additional file 1: Table S1. Phenotypic characteristics of $\mathrm{C} 2, \mathrm{H} 16$ and phylogenetically related $P$. jessenii CIP $105275^{\top}$. (DOCX $59 \mathrm{~kb}$ )

\section{Abbreviations}

BGl: Beijing Genomics Institute; GI: Genomic island; MGE: Mobile genetic elements

\section{Acknowledgments}

This work was supported by the Center for Environmental and Agricultural Microbiology (CREAM) funded by the Villum Foundation.

\section{Authors' contributions}

YQ drafted the manuscript, performed laboratory experiments, and analyzed the data; DW analyzed data; KKB isolated bacteria and assisted in selection of strains, planning and manuscript preparation; CR organized the study and drafted the manuscript. All authors read and approved the final manuscript.

\section{Competing interests}

The authors declare that they have no competing interests.

\section{Author details}

${ }^{1}$ Department of Plant and Environmental Sciences, University of Copenhagen, Frederiksberg, Denmark. ${ }^{2}$ State Key Laboratory of Agricultural Microbiology, College of Life Sciences and Technology, Huazhong Agricultural University, Wuhan, China. ${ }^{3}$ College of Resources and the Environment, Fujian Agriculture and Forestry University, Fuzhou, China. ${ }^{4}$. Craig Venter Institute, La Jolla, CA, USA.

Received: 17 February 2016 Accepted: 4 October 2016

Published online: 03 November 2016

\section{References}

1. Chaturvedi KS, Henderson JP. Pathogenic adaptations to host-derived antibacterial copper. Front Cell Infect Microbiol. 2014;4:3.

2. González-Guerrero M, Raimunda D, Cheng X, Argüello JM. Distinct functional roles of homologous $\mathrm{Cu}^{+}$efflux ATPases in Pseudomonas aeruginosa. Mol Microbiol. 2010;78(5):1246-58.

3. Fu Y, Chang FMJ, Giedroc DP. Copper transport and trafficking at the hostbacterial pathogen interface. Accounts Chem Res. 2014;47(12):3605-13.

4. Porcheron G, Garénaux A, Proulx J, Sabri M, Dozois CM. Iron, copper, zinc, and manganese transport and regulation in pathogenic Enterobacteria: correlations between strains, site of infection and the relative importance of the different metal transport systems for virulence. Front Cell Infect Microbiol. 2013;3:90.

5. Navarro CA, Bernath-von D, Jerez CA. Heavy metal resistance strategies of acidophilic bacteria and their acquisition: importance for biomining and bioremediation. Biol Res. 2013:46(4):363-71.

6. Haas D, Keel C. Regulation of antibiotic production in root-colonizing Pseudomonas spp. and relevance for biological control of plant disease. Annu Rev Phytopathol. 2003;41:117-53.

7. Raaijmakers JM, Vlami M, de Souza JT. Antibiotic production by bacterial biocontrol agents. Antonie Van Leeuwenhoek. 2002;81:537-47.

8. Deora A, Hatano E, Tahara S, Hashidoko Y. Inhibitory effects of furanone metabolites of a rhizobacterium, Pseudomonas jessenii, on phytopathogenic 
Aphanomyces cochlioides and Pythium aphanidermatum. Plant Pathol. 2010; 59:84-99.

9. Berg J, Thorsen MK, Holm PE, Jensen J, Nybroe O, Brandt KK. Cu exposure under field conditions coselects for antibiotic resistance as determined by a novel cultivation-independent bacterial community tolerance assay. Environ Sci Technol. 2010:44(22):8724-8.

10. Strandberg B, Axelsen JA, Pedersen MB, Jensen J, Attrill MJ. Effect of a copper gradient on plant community structure. Environ Toxicol Chem. 2006;25(3):743-53.

11. Thorsen MK, Brandt KK, Nybroe O. Abundance and diversity of culturable Pseudomonas constitute sensitive indicators for adverse long-term copper impacts in soil. Soil Biol Biochem. 2013;57:933-35.

12. Kämpfer $P$, Kroppenstedt RM. Numerical analysis of fatty acid patterns of coryneform bacteria and related taxa. Can J Microbiol. 1996;42:989-1005.

13. Kim OS, Cho YJ, Lee K, Yoon SH, Kim M, Na H, Park SC, Jeon YS, Lee JH, Yi H, Won S, Chun J. Introducing EzTaxon-e: a prokaryotic $16 \mathrm{~S}$ rRNA gene sequence database with phylotypes that represent uncultured species. Int J Syst Evol Microbiol. 2012;62:716-21.

14. Tamura K, Peterson D, Peterson N, Stecher G, Nei M, Kumar S. MEGA5 molecular evolutionary genetics analysis using maximum likelihood, evolutionary distance, and maximum parsimony methods. Mol Biol Evol. 2011;28:2731-39.

15. Field D, Garrity G, Gray T, Morrison N, Selengut J, Sterk P, Tatusova T, Thomson N, Allen MJ, Angiuoli SV, Ashburner M, Axelrod N, Baldauf S, Ballard S, Boore J, Cochrane G, Cole J, Dawyndt P, DeVos P, dePamphilis C, Edwards R, Faruque N, Feldman R, Gilbert J, Gilna P, Glöckner FO, Goldstein $P$, Guralnick R, Haft D, Hancock D. The minimum information about a genome sequence (MIGS) specification. Nat Biotechnol. 2008;26(5):541-7.

16. Brandt KK, Petersen A, Holm PE, Nybroe O. Decreased abundance and diversity of culturable Pseudomonas spp. populations with increasing copper exposure in the sugar beet rhizosphere. FEMS Microbiol Ecol. 2006;56(2):281-91.

17. Bentley DR, Balasubramanian S, Swerdlow HP, Smith GP, Milton J, Brown CG, Hall KP, Evers DJ, Barnes CL, Bignell HR, Boutell JM, Bryant J, Carter RJ, Cheetham RK, Cox AJ, Ellis DJ, Flatbush MR, Gormley NA, Humphray SJ, Irving L, Karbelashvili MS, Kirk SM, Li H, Liu X, Maisinger KS, Murray L, Obradovic B, Ost T, Parkinson ML, Pratt MR, et al. Accurate whole human genome sequencing using reversible terminator chemistry. Nature. 2008; 456(7218):53-9.

18. Yap KP, Gan HM, Teh CS, Baddam R, Chai LC, Kumar N, Tiruvayipati SA, Ahmed $\mathrm{N}$, Thong KL. Genome sequence and comparative pathogenomics analysis of a Salmonella enterica serovar Typhi strain associated with atyphoid carrier in Malaysia. J Bacteriol. 2012;194(21):5970-1.

19. Aziz RK, Bartels D, Best AA, DeJongh M, Disz T, Edwards RA, Formsma K, Gerdes S, Glass EM, Kubal M, Meyer F, Olsen GJ, Olson R, Osterman AL, Overbeek RA, McNeil LK, Paarmann D, Paczian T, Parrello B, Pusch GD, Reich C, Stevens R, Vassieva O, Vonstein V, Wilke A, Zagnitko O. The RAST Server: rapid annotations using subsystems technology. BMC Genomics. 2008;9:75.

20. Stackebrandt E, Ebers J. Taxonomic parameters revisited: tarnished gold standards. Microbiol Today. 2006;33:152-55.

21. Overbeek R, Begley T, Butler RM, Choudhuri JV, Chuang HY, Cohoon M, de Crecy-Lagard V, Diaz N, Disz T, Edwards R, Fonstein M, Frank ED, Gerdes S, Glass EM, Goesmann A, Hanson A, Iwata-Reuyl D, Jensen R, Jamshidi N, Krause L, Kubal M, Larsen N, Linke B, McHardy AC, Meyer F, Neuweger H, Olsen G, Olson R, Osterman A, Portnoy V, et al. The subsystems approach to genome annotation and its use in the project to annotate 1000 genomes. Nucleic Acids Res. 2005;33:5691-702.

22. Lagesen K, Hallin P, Rodland EA, Staerfeldt HH, Rognes T, Ussery DW. RNAmmer: consistent and rapid annotation of ribosomal RNA genes. Nucleic Acids Res. 2007;35:3100-08.

23. Lowe TM, Eddy SR. tRNAscan-SE: a program for improved detection of transfer RNA genes in genomic sequence. Nucleic Acids Res. 1997;25:955-64.

24. Rensing C, Grass G. Escherichia coli mechanisms of copper homeostasis in a changing environment. FEMS Microbiol Rev. 2003;27(2-3):197-213.

25. Fan B, Rosen BP. Biochemical characterization of CopA, the Escherichia coli Cu(l)-translocating P-type ATP-ase. J Biol Chem. 2002;277(49):46987-92.

26. Ekici S, Yang $H$, Koch HG, Daldal F. Novel transporter required for biogenesis of cbb3-type cytochrome c oxidase in Rhodobacter capsulatus. MBio. 2012; 3(1):e00293-11.

27. Zhang XX, Rainey PB. Regulation of copper homeostasis in Pseudomonas Fluorescens SBW25. Environ Microbiol. 2008;10(12):3284-94.
28. Cánovas D, Cases I, de Lorenzo V. Heavy metal tolerance and metal homeostasis in Pseudomonas putida as revealed by complete genome analysis. Environ Microbiol. 2003;5(12):1242-56.

29. Fernández $M$, Udaondo Z, Niqui JL, Duque E, Ramos JL. Synergic role of the two ars operons in arsenic tolerance in Pseudomonas putida KT2440. Environ Microbiol Rep. 2014;6(5):483-89.

30. Jalal S, Ciofu O, Hoiby N, Gotoh N, Wretlind B. Molecular mechanisms of fluoroquinolone resistance in Pseudomonas aeruginosa isolates from cystic fibrosis patients. Antimicrob Agents Chemoter. 2000;44(3):710-12.

31. Wong A, Kassen R. Parallel evolution and local differentiation in quinolone resistance in Pseudomonas aeruginosa. Microbiology. 2011;157(4):937-44.

32. Argüello JM, Raimunda D, Padilla-Benavides T. Mechanisms of copper homeostasis in bacteria. Front Cell Infect Microbiol. 2013;3:73.

33. Bondarczuk K, Piotrowska-Seget Z. Molecular basis of active copper resistance mechanisms in Gram-negative bacteria. Cell Biol Toxicol. 2013; 29(6):397-405

34. Dhillon BK, Chiu TA, Laird MR, Langille MGI, Brinkman FSL. IslandViewer update: improved genomic island discovery and visualization. Nucleic Acids Res. 2013;41(Web Server issue):W129-32.

35. Tribelli PM, Raiger lustman LJ, Catone MV, Di Martino C, Revale S, Méndez BS, López NI. Genome sequence of the polyhydroxybutyrate producer Pseudomonas extremaustralis, a highly stress-resistant antarctic bacterium. J Bacteriol. 2012;194(9):2381-82.

36. Alvarez C, Kukutla P, Jiang JJ, Yu WQ, Xu JN. Draft genome sequence of Pseudomonas sp. Strain Ag1, isolated from the midgut of the malaria mosquito Anopheles gambiae. J Bacteriol. 2012;194(19):5449.

37. Rhodes G, Bosma H, Studholme D, Arnold DL, Jackson RW, Pickup RW. The rulB gene of plasmid pWW0 is a hotspot for the site-specific insertion of integronlike elements found in the chromosomes of environmental Pseudomonas fluorescens group bacteria. Environ Microbiol. 2014;16(8):2374-88.

38. Zhu T, Tian J, Zhang SY, Wu NF, Fan YL. Identification of the transcriptional regulator NcrB in the nickel resistance determinant of Leptospirillum ferriphilum UBK03. Plos one. 2011;6(2):e17367.

39. Zhang WW, Chen LX, Liu DY. Characterization of a marine-isolated mercuryresistant Pseudomonas putida strain SP1 and its potential application in marine mercury reduction. Appl Microbiol Biotechnol. 2012;93(3):1305-14.

40. Woese CR, Kandler O, Wheelis ML. Towards a natural system of organisms: proposal for the domains Archaea, Bacteria, and Eucarya. Proc Natl Acad Sci U S A. 1990;87:4576-79.

41. Garrity GM, Bell JA, Lilburn T. Phylum XIV. Proteobacteria phyl. nov. In: Garrity GM, Brenner DJ, Krieg NR, Staley JT, editors. Bergey's manual of systematic bacteriology, volume 2, part B. 2nd ed. New York: Springer; 2005. p. 1.

42. Validation of publication of new names and new combinations previously effectively published outside the IJSEM. Int J Syst Evol Microbiol. 2005;55(6): 2235-38.

43. Garrity GM, Bell JA, Lilburn T. Class III. Gammaproteobacteria class. nov. In: Garrity GM, Brenner DJ, Krieg NR, Staley JT, editors. Bergey's manual of systematic bacteriology, volume 2, part B. 2nd ed. New York: Springer; 2005. p. 1.

44. Garrity GM, Bell JA, Lilburn T. Order IX. Pseudomonadales. nov. In: Brenner DJ, Krieg NR, Staley JT, Garrity GM, editors. Bergey's manual of systematic bacteriology, volume 2: part B. 2nd ed. New York: Springer; 2005. p. 323.

45. Garrity GM, Bell JA, Lilburn T. Family I. Pseudomonadaceae. In: Brenner DJ, Krieg NR, Staley JT, Garrity GM, editors. Bergey's manual of systematic bacteriology, volume 2: part B. 2nd ed. Springer: New York; 2005. p. 324.

46. Anzai Y, Kim H, Park JY, Wakabayashi H. Phylogenetic affiliation of the pseudomonads based on $16 \mathrm{~S}$ rRNA sequence. Int I Syst Evol Microbiol. 2000; 50(4):1563-89.

47. Palleroni NJ. The road to the taxonomy of Pseudomonas. In: Cornelis $\mathrm{P}$, editor. Pseudomonas: Genomic and Molecular Biology. Norfolk: Caister Academic Press; 2008: p.10.

48. Verhille S, Baida N, Dabboussi F, Izard D, Lecierc H. Taxonomic study of bacteria isolated from natural mineral waters: proposal of Pseudomonas jessenii sp. nov. and Pseudomonas mandelii sp. nov. Syst Appl Microbiol. 1999;22(1):45-58.

49. Ashburner M, Ball CA, Blake JA, Botstein D, Butler H, Cherry JM, Davis AP, Dolinski K, Dwight SS, Eppig JT, Harris MA, Hill DP, Issel-Tarver L, Kasarskis A, Lewis S, Matese JC, Richardson JE, Ringwald M, Rubin GM, Sherlock G. Gene ontology: tool for the unification of biology. The Gene Ontology Consortium. Nat Genet. 2000;25:25-9. 